BULL. AUSTRAL. MATH. SOC.

$80 A 20$

VOL. $7(1972), 312$.

\title{
Transport phenomena in neutral and ionized gases
}

\section{Robert Edward Robson}

Transport processes in gases are examined from both the point of view of non-equilibrium thermodynamics and kinetic theory. Thermodynamics is used to establish concise representations of the general properties of transport coefficients, and in particular, it is shown how the redundancy of Onsager relations in systems with geometrical symmetries can be removed by using the theory of irreducible tensorial sets. The Boltzmann equation for fully and partially ionized gases is solved by a polynomial expansion of the distribution function, and expressions for transport coefficients are obtained in terms of the inverse of certain infinite-dimensional matrices. In the case of fully-ionized gases, it is shown that the approximations associated with the Fokker-Planck equation are accurate as long as the deviation from equilibrium is not substantial. Partiallyionized gases are considered under significantly non-equilibrium conditions brought about by application of an electric field. A new expression is obtained for ion mobility, and comparison is made with the earlier theories of Wannier and Kihara. For electrons, it is shown by way of direct numerical calculations that approximation of the distribution function by the first two terms of an expansion in Legendre polynomials is satisfactory even when the deviation from Maxwellian is substantial. Anisotropic diffusion of ions and electrons in an electric field is also examined, and expressions are derived for longitudinal and transverse diffusion coefficients both via solution of the Boltzmann equation and through thermodynamic analysis.

Received 7 April 1972. Thesis submitted to the Australian National University, February 1972. Degree approved, June 1972. Supervisor: Dr Kailash Kumar. 